\title{
Anti-inflammatory Properties of Fangji Huangqi Tang: Discovery Based on Network Pharmacology, Molecular Docking and Arrowsmith Tools
}

\section{Qingtao Jiang}

Jiangsu Health Vocational College

Lei Han

Jiangsu Provincial Center for Disease Control and Prevention

Xin Liu ( $D$ liux@jscdc.cn )

Jiangsu Provincial Center for Disease Control and Prevention

\section{Feng Zhang}

Jiangsu Provincial Center for Disease Control and Prevention

\section{Research Article}

Keywords: Fangji Huangqi Tang (FHT), Inflammation, Network pharmacology, Molecular docking, Arrowsmith

Posted Date: March 2nd, 2021

DOI: https://doi.org/10.21203/rs.3.rs-242873/v1

License: (c) (i) This work is licensed under a Creative Commons Attribution 4.0 International License. Read Full License 


\section{Abstract}

Fangji Huangqi Tang (FHT) is a classical formula widely used in Chinese clinical practice. In this study, a creative application of FHT for inflammation has been identified by network pharmacology-based framework. Specifically, a total of 17 bioactive compounds including 42 potential targets of FHT, and 205 related targets involved in inflammation were retrieved from mainstream databases and subjected to network analysis. 13 intersection targets indicated the principal elements linked to inflammation therapy. Top terms of Gene Ontology (GO) analysis were identified, while 7 related signaling pathways were revealed by Kyoto Encyclopedia of Genes and Genomes (KEGG) results. Subsequently, Calycosin-PTGS2 with tight binding affinity (BA) was manifested by molecular docking as the critical compound-target couple. Meaningful links between Calycosin and inflammation were implied through Arrowsmith, which overlapped with the findings in enrichment analysis, such as MAPK, NF-KB that could be regulators of PTGS2. In summary, the present study explored the potential targets and signaling pathways of FHT against inflammation, which may help to illustrate the mechanisms responsible for the action of FHT and provide a better understanding of its anti-inflammatory effects.

\section{Introduction}

Fangji Huangqi Tang (FHT) is composed of Stephaniae Tetrandrae Radix (Fangji, FJ), Hedysarum Multijugum Maxim (Huangqi, HQ), Atractylodes Macrocephala Koidz (Baizhu, BZ), Atractylodes Lancea (Thunb.) Dc (Cangzhu, CZ), licorice (Gancao, GC), Radix Angelicae Biseratae (Duhuo, DH) and Coicis Semen (Yiyiren, YYR ${ }^{1-3}$. As a representative prescription for damp clearing of Traditional Chinese medicine (TCM), it possesses the features of Yiqi Qufeng, Lishui and Jianpi, and is usually used for the curation of chronic nephritis, rheumatoid arthritis and osteoarthritis, etc ${ }^{4-6}$. Thus, we speculated that FHT may play roles as an anti-inflammatory formula with broad spectrum.

In current times, researchers did lots of work on the modernization of FHT. Wang et al. developed and validated an ultrahigh performance liquid chromatography method coupled with triple quadrupole mass spectrometry (UHPLC-QqQ-MS) for simultaneous determination of bioactive components in $\mathrm{FHT}^{7-9}$. Chen et al. found that FHT could improve the immunity of mice ${ }^{10}$. The results of these studies laid a good foundation for the application of modern science and technology to explain the rationality and compatibility theory of FHT. But, the depth of these studies was limited to the advanced molecular pharmacological methods, while the theoretical research on effective material has not been really carried out.

Network pharmacology is intensively applied in drug research because of its holistic, comprehensive and efficient characteristics for the systematic study of the internal relationship among 'drugs-targetspathways-diseases' using TCM databases, bioinformatics tools and molecular biology concepts ${ }^{11-13}$. TCM-oriented network pharmacology sheds light on revealing the mechanisms of TCM with multiple compounds through pharmacokinetic evaluation, pathway analysis as well as target prediction, becoming an indispensable method for development of $\mathrm{TCM}^{14-17}$. In many previous studies, network 
pharmacology has successfully unveiled potential targets and pathways of $\operatorname{TCM}^{18-20}$. Meanwhile, molecular docking simulation is frequently employed to further validate the potential bioactive components and core targets ${ }^{21-23}$.

Non-interactive literature-based discovery (NILBD) with Arrowsmith is a practical tactic performing two PubMed searches to retrieve biomedical articles from the Medline database and generating a common list of words and phrases found in the titles of both literatures, which could be utilized to deeply discover the commonness between the subjects that have never been studied ${ }^{24-26}$. The results should be integrated and cross verificated with network pharmacology findings, making the conclusion much more convincing.

In general, this study aims to provide an insight into decoding the possible bioactive compounds and potential mechanism of FHT in inflammation treatment via network pharmacology and Arrowsmith interpretation. Workflow of this work is shown in detail in Figure 1.

\section{Results}

\section{Bioactive compounds' screening of FHT}

FHT formula consists of 7 main herbs: Fangji (FJ), Huangqi (HQ), Baizhu (BZ), Cangzhu (CZ), Gancao (GC), Duohuo (DH) and Yiyiren (YYR). After retrieving from TCMSP with the criteria of $\mathrm{OB} \geq 30 \%$ and DL $\geq 0.18,3,20,7,9,92,9$ and 9 ingredients were obtained for these 7 herbs, respectively. Among them, 11 components were duplicated and removed, resulting in a total of 138 bioactive compounds collected from TCMSP. Next, 306 potentially effective components were also acquired from BATMAN-TCM via a cluster combination (Score cutoff $\geq 20, P$ value $\leq 0.05$ ), followed by generation of co-existed compounds with VENN diagram (https://bioinfogp.cnb.csic.es/tools/venny, Figure S1). At last, 17 compounds from 5 herbs (FJ, HQ, GC, DH and YYR) were identified as putatively bioactive molecules for further study, which are presented in Table 1.

Table 1 A list of the final selected compounds from FHT for network analysis. 


\begin{tabular}{ll}
\hline Compound & PubChem_CID or InChI \\
\hline Tetraneurin A & 174868 \\
Calycosin & 5280448 \\
\hline Isotrifoliol & 5318679 \\
\hline Licocoumarone & 503731 \\
\hline Isoglycyrol & CFWLRXJPRRCJTI-UHFFFAOYSA-N \\
\hline Isolicoflavonol & 5318585 \\
\hline 3'-Methoxyglabridin & 15228663 \\
\hline Gancaonin B & 5317479 \\
\hline Gancaonin A & 5317478 \\
\hline Lupiwighteone & 5317480 \\
\hline Glycyrin & 480787 \\
\hline Glyzaglabrin & 5317777 \\
\hline Licoricone & 5319013 \\
\hline Glycyrol & 5320083 \\
\hline Angelol D & 44144314 \\
Angelicone & 616303 \\
\hline Coixenolide & DPQCZNIGGNJGTD-FLZCLMPRSA-N \\
\hline
\end{tabular}

\section{Putative targets of FHT and construction of C-T network}

240 putative targets were inferred by an alliance of TCMSP and BATMAN-TCM. After removing duplicates, 43 potential targets linked to 16 compounds of FHT were enrolled. PPI was derived from STRING with a combined score $\geq 0.90$, creating a network consisted of 42 molecules without PRSS1 that was unconnected mutually (Figure 2A). Then, a visual C-T network of FHT with 58 nodes and 392 edges was established by Cytoscape 3.6.0 (Figure 2B). 3'-Methoxyglabridin, Gancaonin B, Calycosin, Lupiwighteone, and Gancaonin A were top 5 bioactive compounds with maximum degree in network.

\section{Functional annotation of FHT targets}

To investigate the potential pharmacological mechanism of FHT, we analyzed GO term and KEGG pathway by DAVID. The top 10 significant GOBP, GOCC and GOMF terms $(P \leq 0.05)$ based on counts were listed in Figure $3 \mathrm{~A}-3 \mathrm{C}$, respectively. The BPs of FHT targets may relate to 'positive regulation of transcription from RNA polymerase II promoter', 'transcription, DNA-templated', 'signal transduction', and so on. GOCC analysis showed that 'nucleus', 'plasma membrane' and 'cytoplasm' accounted for the top 3 proportion (28, 26, and 19 targets, respectively). Besides, 'protein binding', 'enzyme binding' and 'ATP binding' were mainly enriched terms indicated by GOMF. In addition, 34 KEGG pathways were recognized as $P<0.05$. Top 10 enrichment results were shown in Figure 3D, delineating the critical KEGG pathways (including Cancer, Calcium, and cAMP signaling pathways, etc.).

\section{T-D network construction and Common targets discovery}

205 targets related to inflammation were gained through a double screening of TTD and BATMAN-TCM. Based on previous strategy, STRING database was employed to reveal the interactions among disease targets (Figure 4A). The T-D network of inflammation with 205 nodes and 2993 edges was visualized by 
Cytoscape 3.6.0 (Figure 4B). IL-6, TNF, IL-10, ALB and TLR4 are top 5 predominant targets with maximum degree in network. At last, on account of 42 putative targets of FHT and 205 targets related to inflammation, 13 common targets were screened out by intersection. Active compounds associated with selected overlapping targets are listed in Table 2.

Table 2 Details of 13 inflammation-related targets of FHT via Uniprot.

\begin{tabular}{|l|l|l|l|}
\hline Gene & & Uniprot & Compound \\
\hline ESR1 & Estrogen receptor & P03372 & Calycosin \\
\hline AR & Androgen receptor & P10275 & Calycosin \\
\hline GABRA1 & $\begin{array}{l}\text { Gamma-aminobutyric acid receptor subunit } \\
\text { alpha-1 P14867 }\end{array}$ & Angelicone \\
\hline ACHE & Acetylcholinesterase & P22303 & $\begin{array}{l}3^{\prime}- \\
\text { Methoxyglabridin }\end{array}$ \\
\hline PTGS1 & Prostaglandin G/H synthase 1 & P23219 & Calycosin \\
\hline NOS2 & Nitric oxide synthase, inducible & P35228 & Calycosin \\
\hline PTGS2 & Prostaglandin G/H synthase 2 & P35354 & Calycosin \\
\hline PPARG & $\begin{array}{l}\text { Peroxisome proliferator activated receptor } \\
\text { gamma }\end{array}$ & P37231 & Calycosin \\
\hline MAPK14 & Mitogen-activated protein kinase 14 & Q16539 & Calycosin \\
\hline NR3C1 & Glucocorticoid receptor & P04150 & Coixenolide \\
\hline ESR2 & Estrogen receptor beta & Q92731 & Calycosin \\
\hline TOP2A & DNA topoisomerase IIA & P11388 & $\begin{array}{l}3^{\prime}- \\
\text { Methoxyglabridin }\end{array}$ \\
\hline TOP2B & DNA topoisomerase IIB & Q02880 & $\begin{array}{l}3^{\prime}- \\
\text { Methoxyglabridin }\end{array}$ \\
\hline
\end{tabular}

\section{Functional enrichment analysis of common targets}

To further validate whether the candidate targets as mentioned above was correlated with FHT against inflammation, functional annotation was performed via DAVID. Interestingly, the results were similar to that of FHT targets, indicating the main function of FHT was to treat inflammation. Ultimately, 29 BPs, 7 CCs and $25 \mathrm{MFs}$ enriched for these targets had a $P$ value less than 0.05 . The representative results were shown in Figure 5A-5C. Meanwhile, in terms of KEGG, a total of 7 pathways (Figure 5D) were screened, including 'Pathways in cancer', 'Leishmaniasis', 'Prolactin signaling pathway' and 'Retrograde endocannabinoid signaling' when corrected by a $P$ value $\leq 0.01$.

\section{Major bioactive compound-target and mechanism speculation}

To explore the potential bioactive components and targets of FHT more accurately, a compound-targetfunction module was built by combing the networks above. Based on topology to find densely connected protein complexes, MCODE divided C-T network into 4 function modules (Figure 6). The cluster with highest MCODE Score (10.471) had 18 nodes and 89 edges, verifying PTGS2, TOP2A, NR3C1 as main targets, while Calycosin as a pivotal compound.

Molecular docking technology was applied to verify the affinity between Calycosin and the crystal structure of PTGS2 which was recognized as the most promising pair of compound-target. The obtained 
docking result indicates the receptor-ligand interaction. According to Figure 7, Calycosin small molecule forms hydrogen bonds with GLU524 residue on PTGS2. The BA in this couple was $-8.1 \mathrm{~kJ} / \mathrm{mol}$, which was less than that of Dihomo-gamma-linolenic acid used as a positive control $(-6.2 \mathrm{~kJ} / \mathrm{mol})$, further proving the strong binding ability of Calycosin to PTGS2.

No published data concerning Calycosin and inflammation could be retrieved through PubMed. Thus, meaningful links were further implied by Arrowsmith through a two-step query, yielding a list containing terms that appeared in both Calycosin and inflammation literature. Finally, there were 105 terms on the current list ( 26 are predicted to be relevant with Probability $\geq 0.33$ ), which was shown ranked according to predicted relevance (Table S1). There was a consistency to the findings in enrichment analysis, such as MAPK, NF-KB involved in 'Pathways of cancer' and 'VEGF signaling pathway'.

\section{Discussion}

Inflammation is a chronic and progressive pathogen or immune-mediated pathological process causing consecutive symptoms including pain, fever, fatigue and so on ${ }^{27}$. Despite a positive defense reaction, dysregulated inflammation could eventually cause disorders and conditions, such as rheumatoid arthritis, atherosclerosis, periodontitis, and even cancers ${ }^{28}$.

Widely applied therapeutic agents include antibiotics, non-steroidal anti-inflammatory drugs (NSAIDs) and glucocorticoids, which have achieved good therapeutic effects ${ }^{29}$. However, some side effects have resulted because of their extensive use ${ }^{30}$. Meanwhile, based on continuous breakthroughs in studies for compatibility of TCM, increasing attention has been paid because of its significant therapeutic effects and reduced side effects ${ }^{31}$.

A series of clinical practices demonstrated that FHT could be used to treat acute or chronic glomerulonephritis, rheumatoid arthritis and ankylosing spondylitis, relieving typical symptoms as well as suppressing inflammatory reaction ${ }^{4}$. By observing the effects of FHT on immune function of normal mice, it was found that the immunoregulatory activity of FHT was significant ${ }^{10,32}$.

Although researches on the treatment of inflammation with FHT continue, no systematic and comprehensive understanding of the relationships between targets and pathways involved has emerged. Fortunately, network pharmacology, which is especially suitable for multi-compound and multi-target analysis, provides a prospective method to solve this problem with bioinformatics, molecular biology and databases ${ }^{33-35}$.

In current study, we predicted and analyzed the potential mechanisms of FHT via systematic network pharmacology tools. After preliminary screening based on databases in combination with ADME properties, 17 bioactive ingredients of FHT were disclosed. Thereafter, 43 candidate targets from 16 potential compounds were assembled for further analysis. Ranked by connectivity, 3'-Methoxyglabridin, Gancaonin B, Calycosin were the top 3 bioactive compounds with the effect of mitigating inflammations, while PTGS2, ESR1, NOS2 were the top 3 targets frequently connected with inflammation-related 
pathways, such as NF-KB and PI3K/AKT. These results suggested that FHT formula could probably regulate anti-inflammatory action and the immune system.

Subsequently, 13 common targets were screened out by intersecting from C-T and T-D networks, among which 8 genes were derivated from Calycosin. Functional enrichment analyses suggested these targets were closely related with inflammatory- and immune-related molecules or signaling pathway. For example, 'Pathways in cancer' and 'cAMP signaling pathway' precisely implied the extensive characteristics of inflammation and its potential harm of carcinogenesis. Overall, PPI and constructed networks indicated that the therapeutic effects of FHT on inflammation may be achieved through the synergistic and additive effects on multiple molecules and multiple pathways.

Finally, module analysis uncovered the most promising cluster. Calycosin-PTGS2 was recognized as the pivotal compound-target couple. Then, molecular docking technology was utilized to verify the interaction of PTGS2 with Calycosin. The results showed that the binding free energy of Calycosin with the core target was much less than $-5 \mathrm{~kJ} / \mathrm{mol}$, which indicated that the ligand molecule could spontaneously bind to the receptor protein and had a strong binding force ${ }^{36}$. Finally, we put Calycosin and inflammation into Arrowsmith, generating a string of terms consisted of NF-KB, MAPK, etc., which was consistent with what we found in the enrichment analysis. Reportedly, several inflammatory stimuli have been shown to affect PTGS2 expression in a NF-KB or ERK dependent manner, which implies the underlying role of CalycosinNF-KB/MAPK-PTGS2 axis in FHT treatment against inflammation ${ }^{37,38}$.

\section{Conclusion}

In general, our study was noteworthy that network pharmacology measures definitely shed light on investigating the complicated action of FHT. Certain limitations also existed firstly due to lack of animal or in vitro experiments. Second, in light of the difficulty in data acquisition, certain databases may not include all known or unknown targets and PPI. This situation could be improved in the future when more data were available.

\section{Materials And Methods}

\section{Identification of bioactive ingredients}

The ingredients of FHT formula containing Stephaniae Tetrandrae Radix (FJ), Hedysarum Multijugum Maxim (HQ), Atractylodes Macrocephala Koidz (BZ), Atractylodes Lancea (Thunb.)Dc (CZ), licorice (GC), Radix Angelicae Biseratae (DH) and Coicis Semen (YYR) were firstly retrieved from TCMSP database (http://lsp.nwu.edu.cn/tcmsp.php) ${ }^{39}$. Oral bioavailability $(\mathrm{OB}) \geq 30 \%$ and drug likeness $(\mathrm{DL}) \geq 0.18$ were used to screen involved ingredients, which are the most important pharmacokinetic parameters for estimating drug ability. Subsequently, the bioactive components of FHT were also obtained from BATMAN-TCM (http://bionet.ncpsb.org/batman-tcm/) via a cluster combination, followed by generation of co-existed compounds with previous step ${ }^{40}$. 


\section{Prediction of the candidate targets of bioactive compounds}

The selected compounds were appointed for targets exploration. Potential drug targets were identified through Traditional Chinese Medicine Systems Pharmacology (TCMSP) and Bioinformatics Analysis Tool for Molecular Mechanism of Traditional Chinese Medicine (BATMAN-TCM) by a similarity-based method. Thereafter, candidate targets in common were mapped to Uniprot (http://www.uniprot.org/) for annotation and normalization.

\section{Prediction of targets for inflammation treatment}

The targets related to inflammation were predicted by integrating multisource databases, including Therapeutic Target Database (TTD, http://db.idrblab.net/ttd/) and Online Mendelian Inheritance in Man (OMIM, http://www.omim.org/). These two databases illustrate the relationship between targets and disease from diverse perspectives. TTD is comprehensive and freely available, from which the user can obtain information in form of disease-target-drug on the known and explored therapeutic protein and nucleic acid targets and the corresponding drugs ${ }^{41}$. OMIM focuses on the relationship between phenotype and genotype on all known mendelian disorders and over 15,000 genes ${ }^{42}$. By searching for items with suffix 'itis' or containing 'inflammation'/'inflammatory' among the downloaded file 'Targets to disease mapping with ICD identifiers' or under Gene Map, the targets were retrieved and integrated via TTD and OMIM, respectively.

\section{Protein-Protein Interaction (PPI) data collection}

Search Tool for the Retrieval of Interacting Genes (STRING, https://string-db.org/) was employed to collect possible PPI by uploading putative targets that related to inflammation and active compounds, respectively ${ }^{43}$. Species was restricted to 'Homo sapiens' with a confidence score $>0.90$. The interactions among nodes with layout degrees could be inferred for further network construction.

\section{Network construction and analysis}

Two kinds of networks in this study were established using Cytoscape 3.6 .0 software: compound-target network (C-T network) and target-disease network (T-D network) ${ }^{44}$. C-T network was composed of bioactive compounds and their potential targets, which was built to reveal the drug-target interactions. TD network was built based on the potential targets and their related diseases. In the networks, the nodes represented compounds and targets, while the edges stood for the interactions between two nodes. Furthermore, the significance of each node in the networks was assessed via one crucial topological parameter 'degree', which was defined as the total of edges associated with a node. The protein complexes present as clusters were analyzed via plugin MCODE 1.6 of Cytoscape 3.6.0.

\section{Functional Enrichment Analyses}

GO terms of biological process (GOBP), cellular component (GOCC) and molecular function (GOMF) as well as KEGG pathways of the candidate targets deduced from bioactive compounds and inflammation 
were analyzed via the (DAVID) database ${ }^{45}$. The enrichment results reflecting top items ranked by gene counts were downloaded. Then, R software 3.6.1 was applied for data visualization with 'ggplot2' package.

\section{Molecular Docking}

Molecular docking is used to verify the binding activity between target proteins and active components. The protein structure of the key target was obtained from the RCSB PDB database (https://www.rcsb.org/), and the 3D structure of the pivotal compound was obtained from the ZINC database (http://zinc.docking.org/) ${ }^{46}$. Discovery Studio 2016 and PyRx 0.9.8 was used to hydrogenate the protein, find active pockets, and conduct molecular docking verification with $\mathrm{FHD}^{47}$. The total-score function of AutoDock/Vina module was used to score the interaction between active components and target proteins. The visualization of binding geometry and interaction was present via PyMol 1.7. $\mathrm{x}^{48}$.

\section{Potential mechanism discovery by NILBD}

NILBD was conducted by Arrowsmith (http://arrowsmith.psych.uic.edu/) for deep validation of the pharmacological mechanism on key component of $\mathrm{FHT}^{25,49,50}$. Information retrieval with keywords was done on a two-node search interface, looking for links that may be present in common between two distinct sets of articles in MEDLINE. The first search defines 'literature A' and the second defines 'literature $C^{\prime}$. The program then generates a "B-list" of words and phrases found in the titles of both literatures, which assisted us exploring a crucial molecule or purported mechanism involved in the role of FHT on inflammation.

\section{Data availability}

The datasets generated during and/or analyzed during the current study are available from the corresponding author on reasonable request.

\section{Declarations}

\section{Acknowledgments}

This work was supported by Qinglan Project of Jiangsu Province, Jiangsu Provincial 333 Project (No. BRA2018398), Natural Science Foundation of Jiangsu (No. BK20201485), Key medical research project of Jiangsu Health Commission (No. K2019026) and Jiangsu Provincial Medical Youth Talent (No. QNRC2016549).

\section{Author contributions}

X.L. and F.Z. conceived and designed the experiments; Q.T.J. and L.H. performed the research; both Q.T.J. and X.L. wrote the manuscript with input from all authors and supervised the findings of this work. All authors read and approved the final manuscript. 


\section{Competing interests}

The authors report no conflicts of interest regarding this work.

\section{References}

1. Zhang, W. N., Yang, L., He, S. S., Qin, X. M. \& Li, A. P. Metabolomics coupled with integrative pharmacology reveal the protective effect of FangjiHuangqi Decoction against adriamycin-induced rat nephropathy model. J Pharm Biomed Anal 174, 525-533 (2019).

2. Yang, L. et al. Comprehensive investigation of mechanism and effective ingredients of Fangji Huangqi Tang by serum pharmacochemistry and network pharmacology. Biomed Chromatogr 34, e4785 (2020).

3. Liu, X. et al. Study on spectrum-effect correlation for screening the effective components in Fangji Huangqi Tang basing on ultra-high performance liquid chromatography-mass spectrometry. Phytomedicine 47, 81-92 (2018).

4. Zhang, J. J. \& Yu, R. H. Treatment of focal segmental glomerular sclerosis by modified fangji huangqi decoction: a case report of one patient. Zhongguo Zhong Xi Yi Jie He Za Zhi 35, 252-253 (2015).

5. Wang, R., Ma, T. M., Liu, F. \& Gao, H. Q. Research progress on pharmacological action and clinical application of Stephania Tetrandrae Radix. Zhongguo Zhong Yao Za Zhi 42, 634-639 (2017).

6. Liu, X., Zhou, Q. G., Zhu, X. C., Xie, L. \& Cai, B. C. Screening for Potential Active Components of Fangji Huangqi Tang on the Treatment of Nephrotic Syndrome by Using Integrated Metabolomics Based on "Correlations Between Chemical and Metabolic Profiles". Front Pharmacol 10, 1261 (2019).

7. Wang, X. et al. Ultra high performance liquid chromatography with tandem mass spectrometry method for the determination of tetrandrine and fangchinoline in rat plasma after oral administration of Fangji Huangqi Tang and Stephania tetrandra S. Moore extracts. J Sep Sci 38, 1286-1293 (2015).

8. Wang, X. et al. Screening and identification of multiple constituents and their metabolites of Fangji Huangqi Tang in rats by ultra-high performance liquid chromatography coupled with quadrupole time-of-flight tandem mass spectrometry basing on coupling data processing techniques. $J$ Chromatogr B Analyt Technol Biomed Life Sci 985, 14-28 (2015).

9. Wang, X., Liu, X., Zhu, T. \& Cai, B. Development and Validation of an UHPLC-QqQ-MS Technique for Simultaneous Determination of Ten Bioactive Components in Fangji Huangqi Tang. J Anal Methods Chem 2016, 1435106 (2016).

10. Chen HY, Jie LQ \& YJ, W. Effect of Fangji Huangqi Tang reduction intervention on the expression of MCP-1 in the renal tissue of adriamycin nephropathy rats. Chinese journal of traditional Chinese and western medicine combined with kidney disease 20, 958-962 (2011).

11. Hopkins, A. L. Network pharmacology: the next paradigm in drug discovery. Nat Chem Biol 4, 682-690 (2008).

12. Hopkins, A. L. Network pharmacology. Nat Biotechnol 25, 1110-1111 (2007). 
13. Hao, D. C. \& Xiao, P. G. Network pharmacology: a Rosetta Stone for traditional Chinese medicine. Drug Develop Res 75, 299-312 (2014).

14. Zhang, G. B., Li, Q.-y., Chen, Q.-I. \& Su, S.-b. Network Pharmacology: A New Approach for Chinese Herbal Medicine Research. Evid Based Complement Alternat Med 2013, 621423 (2013).

15. Zhang, R. Z., Zhu, X., Bai, H. \& Kang, N. Network Pharmacology Databases for Traditional Chinese Medicine: Review and Assessment. Front Pharmacol 10 (2019).

16. Li, J. et al. Traditional Chinese Medicine-Based Network Pharmacology Could Lead to New Multicompound Drug Discovery. Evid Based Complement Alternat Med 2012, 149762 (2012).

17. Kibble, M. et al. Network pharmacology applications to map the unexplored target space and therapeutic potential of natural products. Nat Prod Rep 32, 1249-1266 (2015).

18. Wei, S. et al. Network pharmacology exploration reveals the bioactive compounds and molecular mechanisms of Li-Ru-Kang against hyperplasia of mammary gland. Mol Genet Genomics 294, 11591171 (2019).

19. Li, J. Y., Chen, H. Y., Dai, W. J., Lv, Q. J. \& Chen, C. Y. Artificial Intelligence Approach To Investigate the Longevity Drug. J Phys Chem Lett 10, 4947-4961 (2019).

20. Li, R. et al. Network Pharmacology and bioinformatics analyses identify intersection genes of niacin and COVID-19 as potential therapeutic targets. Brief Bioinform (2020).

21. Kolb, P., Ferreira, R. S., Irwin, J. J. \& Shoichet, B. K. Docking and chemoinformatic screens for new ligands and targets. Curr Opin Biotechnol 20, 429-436 (2009).

22. Pinzi, L. \& Rastelli, G. Molecular Docking: Shifting Paradigms in Drug Discovery. Int J Mo/ Sci 20 (2019).

23. Kaur, T., Madgulkar, A., Bhalekar, M. \& Asgaonkar, K. Molecular Docking in Formulation and Development. Curr Drug Discov Technol 16, 30-39 (2019).

24. Hsih-Te, Y., Jiun-Huang, J., Yue-Ting, W., llya, S. \& Jung-Hsien, C. Literature-based discovery of new candidates for drug repurposing. Brief Bioinform 18, 488-497 (2016).

25. Hristovski, D., Peterlin, B., Mitchell, J. A. \& Humphrey, S. M. Using literature-based discovery to identify disease candidate genes. Int J Med Inform 74, 289-298 (2005).

26. Preiss, J. \& Stevenson, M. Quantifying and filtering knowledge generated by literature based discovery. BMC Bioinformatics 18, 249 (2017).

27. Carl, N. Points of control in inflammation. Nature 420, 846-852 (2002).

28. Diakos, C. I., Charles, K. A., McMillan, D. C. \& Clarke, S. J. Cancer-related inflammation and treatment effectiveness. Lancet Oncol 15, e493-503 (2014).

29. Simpson, S. J. Interfering with Inflammation. Sci Signal 1, ec51 (2008).

30. González-Gay, M. A. \& González-Juanatey, C. Inflammation: NSAIDs and cardiovascular risk in arthritis. Nat Rev Cardio/ 14, 69-70 (2017).

31. Li, S. M., Li, J. G. \& Xu, H. A New Perspective for Chinese Medicine Intervention for Coronary Artery Disease: Targeting Inflammation. Chin J Integr Med 25, 3-8 (2019). 
32. Li, J., Zhang, F. \& Li, J. The Immunoregulatory Effects of Traditional Chinese Medicine on Treatment of Asthma or Asthmatic Inflammation. Am J Chinese Med 43, 1059-1081 (2015).

33. Gosak, M. et al. Network science of biological systems at different scales: A review. Phys Life Rev 24, 118-135 (2018).

34. Medina-Franco, J. L., Giulianotti, M. A., Welmaker, G. S. \& Houghten, R. A. Shifting from the single to the multitarget paradigm in drug discovery. Drug Discov Today 18, 495-501 (2013).

35. Janga, S. C. \& Tzakos, A. Structure and organization of drug-target networks: insights from genomic approaches for drug discovery. Mol Biosyst 5, 1536-1548 (2009).

36. Dallakyan, S. \& Olson, A. J. Small-molecule library screening by docking with PyRx. Methods Mol Biol 1263, 243-250 (2015).

37. Harvey, A. E. et al. Calorie Restriction Decreases Murine and Human Pancreatic Tumor Cell Growth, Nuclear Factor-кB Activation, and Inflammation-Related Gene Expression in an Insulin-like Growth Factor-1 Dependent Manner. Plos One 9, e94151 (2014).

38. Santulli, P. et al. Role of the protein kinase BRAF in the pathogenesis of endometriosis. Expert Opin Ther Targets 20, 1017-1029 (2016).

39. Ru, J. et al. TCMSP: a database of systems pharmacology for drug discovery from herbal medicines. J Cheminform 6, 13 (2014).

40. Liu, Z., Guo, F., Wang, Y., Li, C. \& He, F. BATMAN-TCM: a Bioinformatics Analysis Tool for Molecular mechanism of Traditional Chinese Medicine. Sci Rep 6, 21146 (2016).

41. Yunxia, W. et al. Therapeutic target database 2020: enriched resource for facilitating research and early development of targeted therapeutics. Nucleic Acids Res 48, D1031-D1041 (2020).

42. Xu, J. \& Li, Y. Discovering disease-genes by topological features in human protein-protein interaction network. Bioinformatics 22, 2800-2805 (2006).

43. Szklarczyk, D. et al. STRING v11: protein-protein association networks with increased coverage, supporting functional discovery in genome-wide experimental datasets. Nucleic Acids Res 47, D607D613 (2019).

44. Shen, Y. et al. Network Pharmacology-Based Analysis of Xiao-Xu-Ming Decoction on the Treatment of Alzheimer's Disease. Front Pharmacol 11, 595254 (2020).

45. Jiao, X. et al. DAVID-WS: a stateful web service to facilitate gene/protein list analysis. Bioinformatics 28, 1805-1806 (2012).

46. Liu, F. et al. Study on mechanism of matrine in treatment of COVID-19 combined with liver injury by network pharmacology and molecular docking technology. Drug Deliv 28, 325-342 (2021).

47. Zheng, W. J. et al. Examining the effector mechanisms of Xuebijing injection on COVID-19 based on network pharmacology. BioData Min 13, 17 (2020).

48. Nie, C. D. et al. Study on molecular of anti-tumor mechanism of Forsythia suspensa based on molecular docking and network pharmacology. Zhongguo Zhong Yao Za Zhi 45, 4455-4465 (2020). 
49. Swanson, D. R. Literature-based Resurrection of Neglected Medical Discoveries. J Biomed Discov Collab 6, 34-47 (2011).

50. Kostoff, R. N. Validating discovery in literature-based discovery. J Biomed Inform 40, 448-450; author reply 450-442 (2007).

\section{Figures}

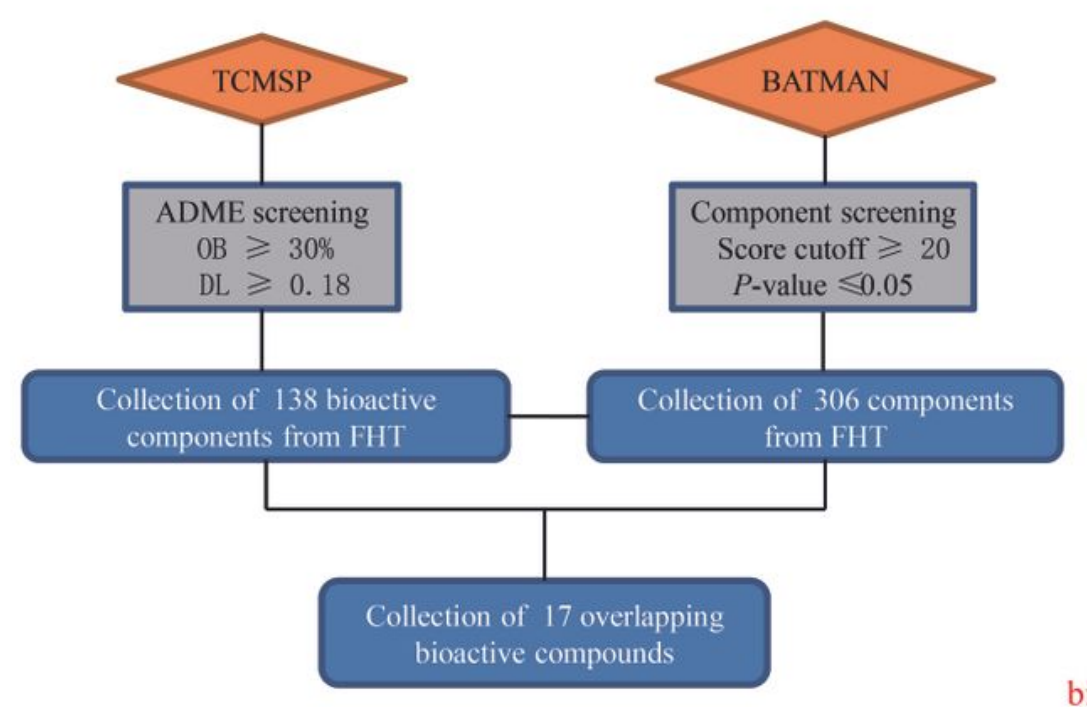

Step 1:

collection of

bioactive compounds

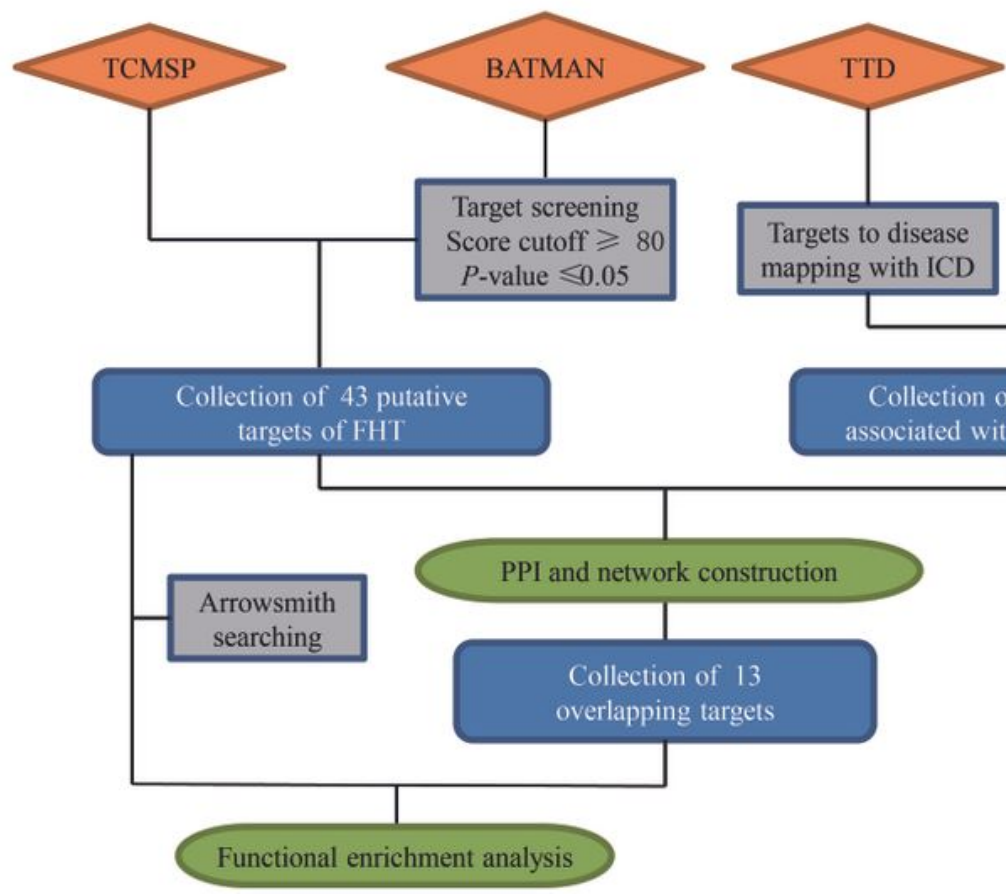

Step 2:

collection of targets related to FHT and inflammation and network analysis 
Figure 1

Workflow of network pharmacology analysis of FHT on inflammation.

A

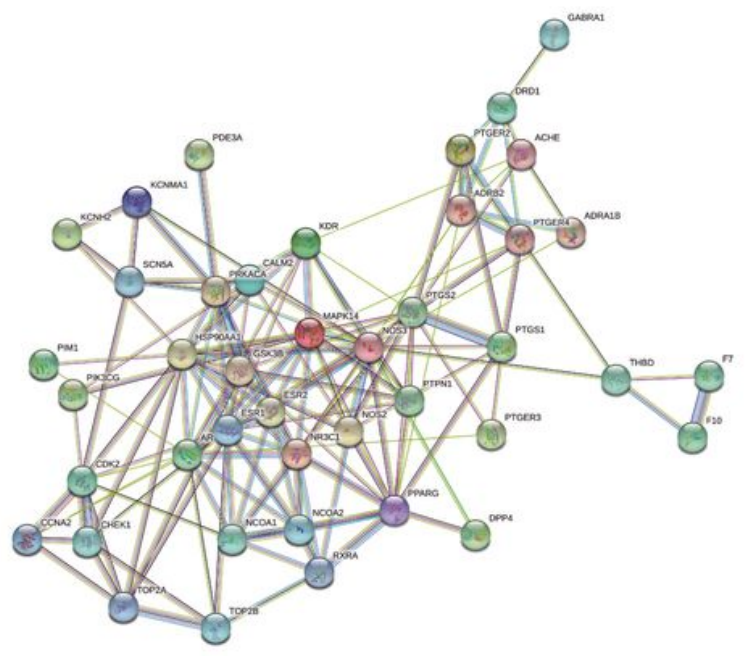

B

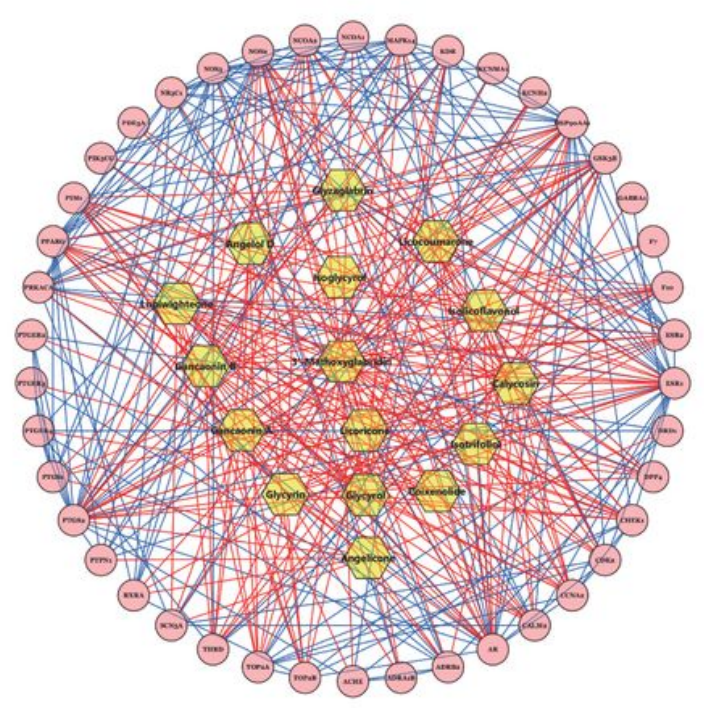

Figure 2

Construction of PPI and C-T network on putative targets of FHT. (A) PPI of 42 potential targets from FHT via STRING database. (B) C-T network constructed by Cytoscape. Yellow nodes stand for bioactive compounds and pink nodes stand for putative targets. 
A

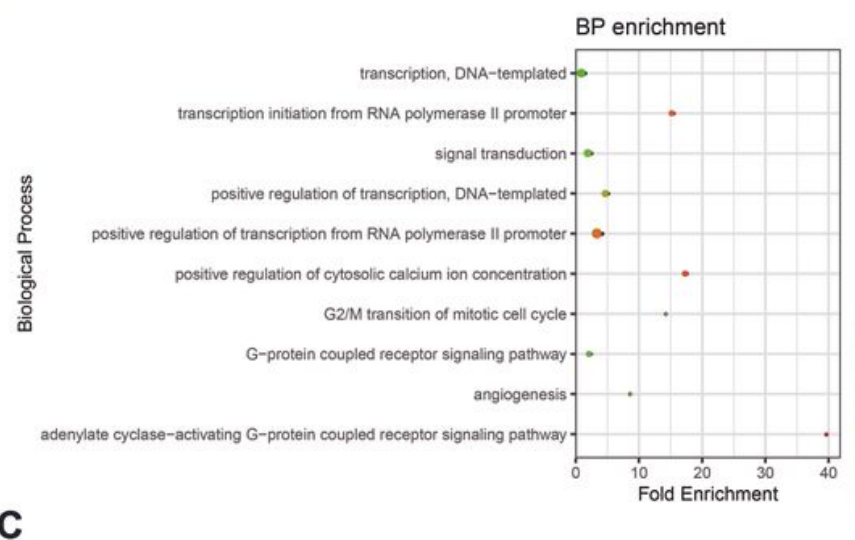

C

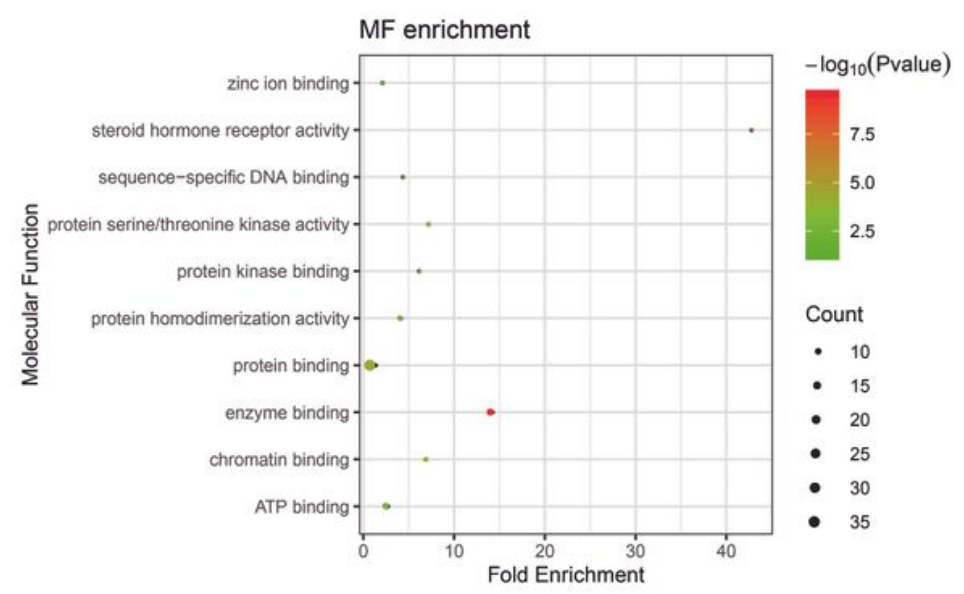

B
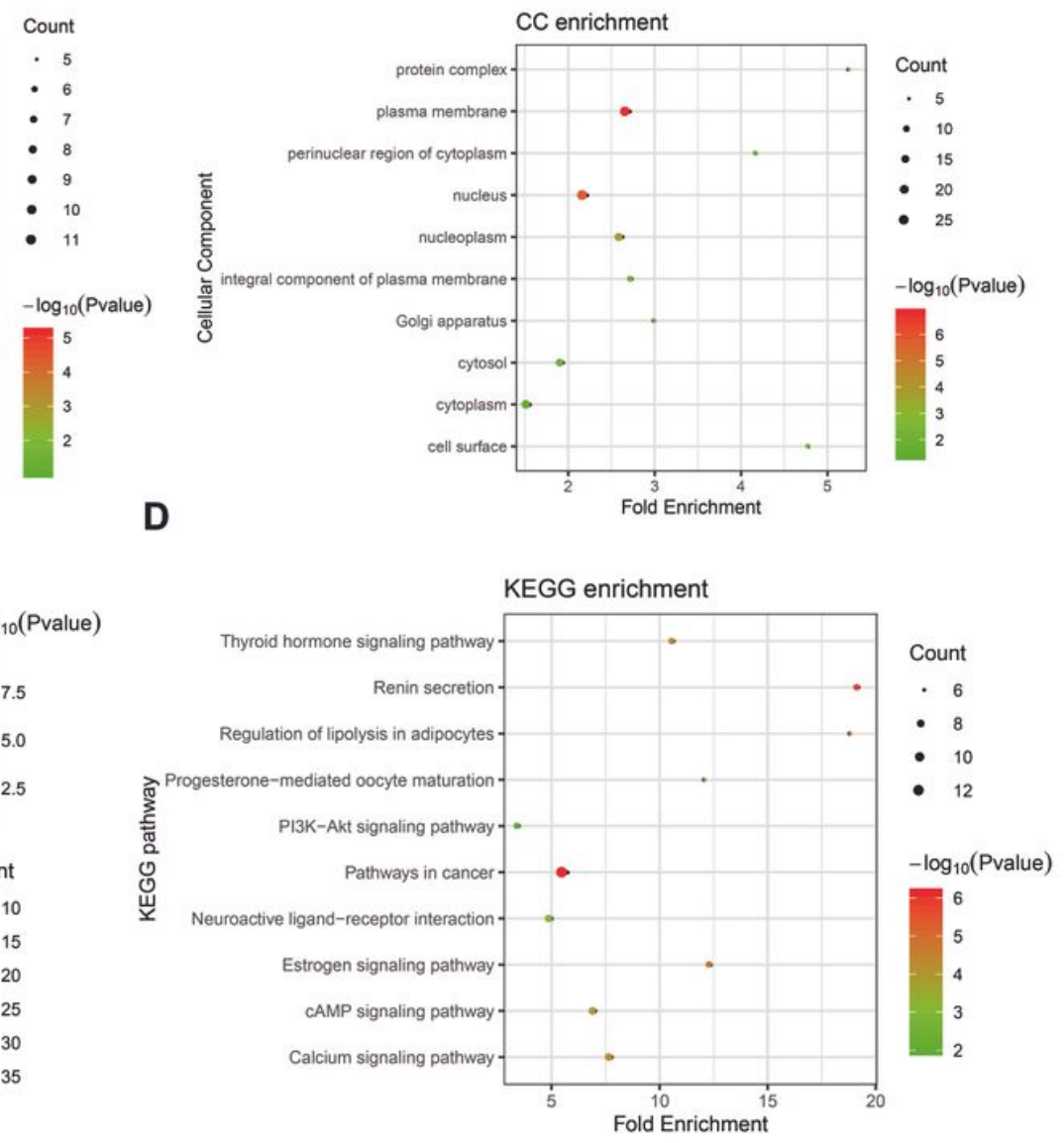

\section{Figure 3}

Enrichment analysis of FHT targets. (A) GO enrichment of BP. (B) (A) GO enrichment of CC. (C) GO enrichment of MF. (D) KEGG pathway enrichment.

A

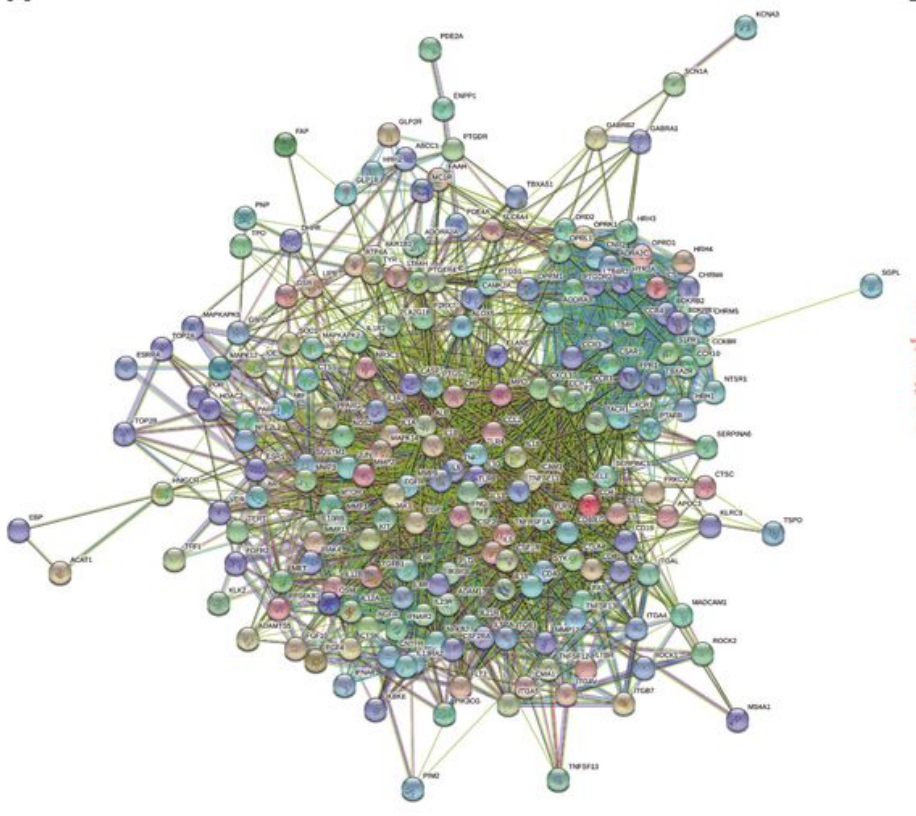

B

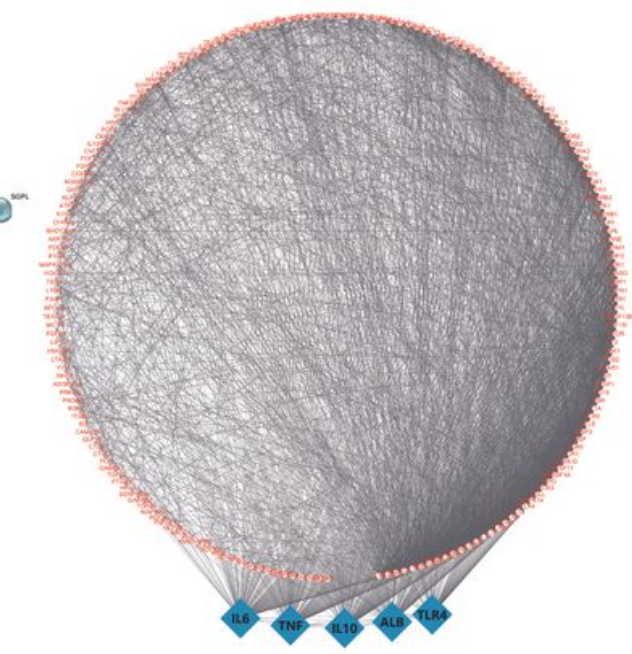

Figure 4 
Construction of PPI and T-D network on potential targets of inflammation. (A) PPI of 42 potential targets deduced from inflammation via STRING database. (B) T-D network constructed by Cytoscape. Red nodes stand for putative disease targets.

A

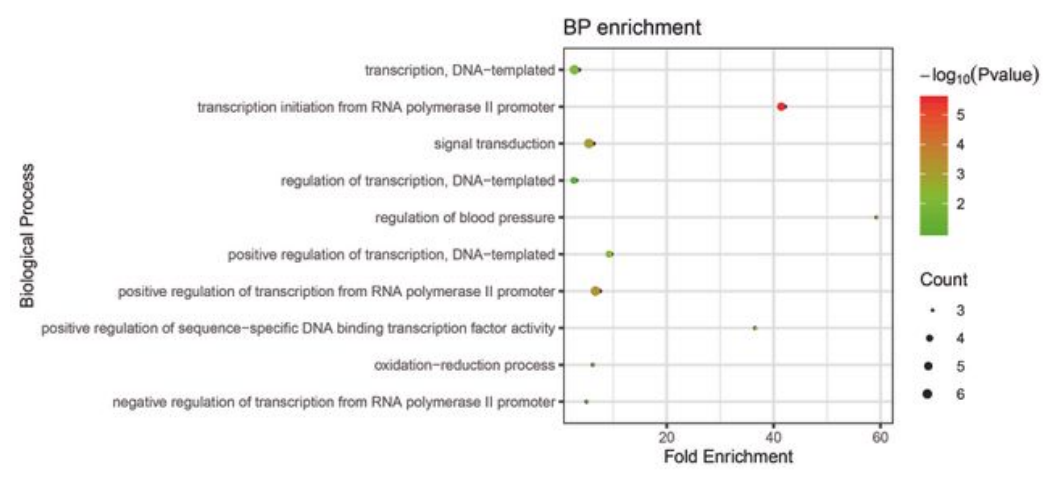

C

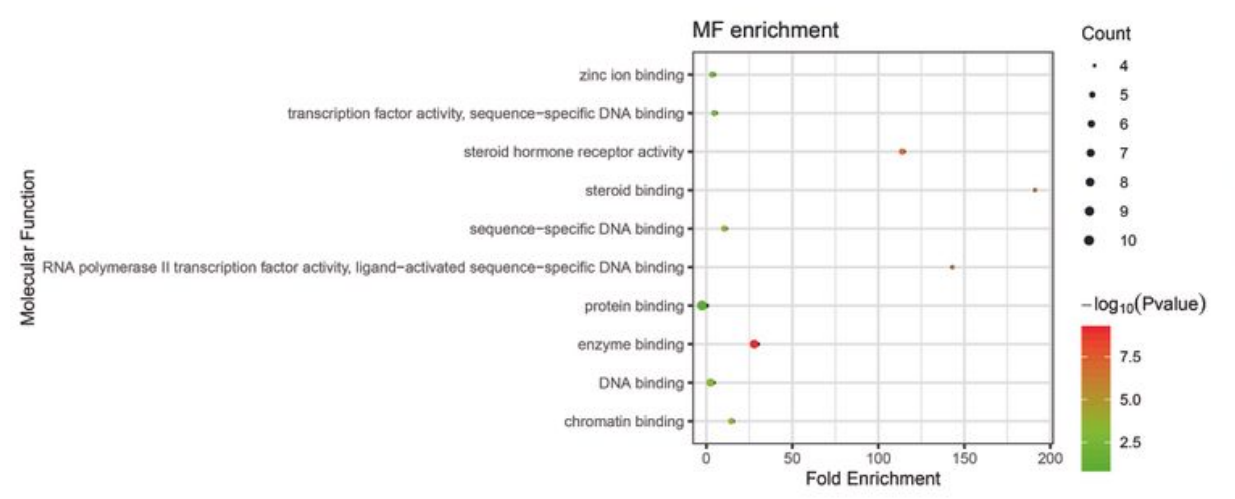

Figure 5

Enrichment analysis of common targets. (A) GO enrichment of BP. (B) (A) GO enrichment of CC. (C) GO enrichment of MF. (D) KEGG pathway enrichment.
B

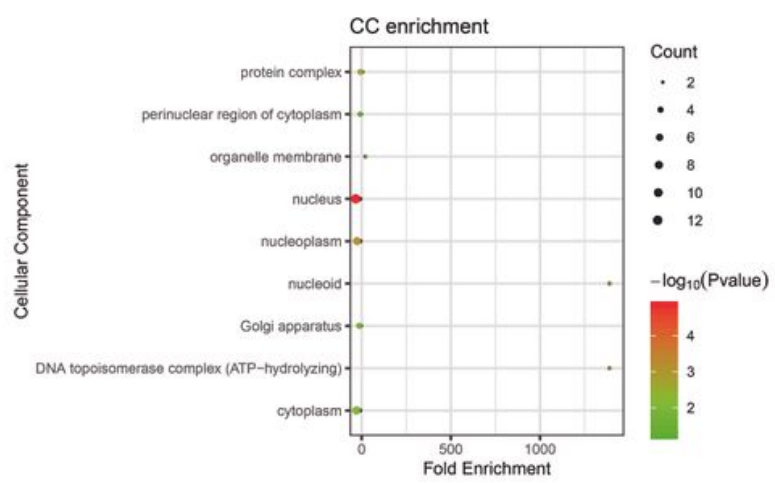

D

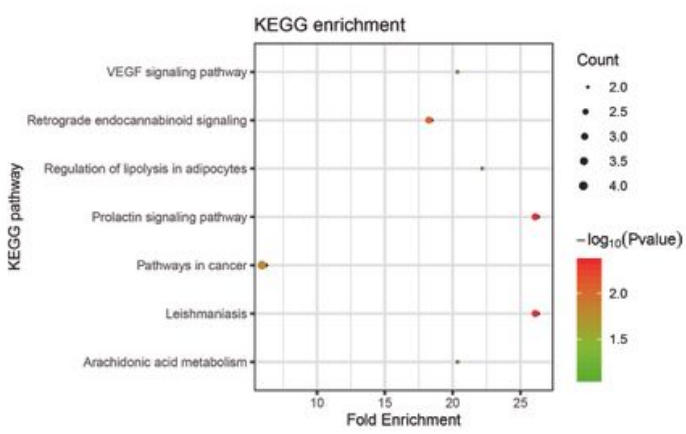




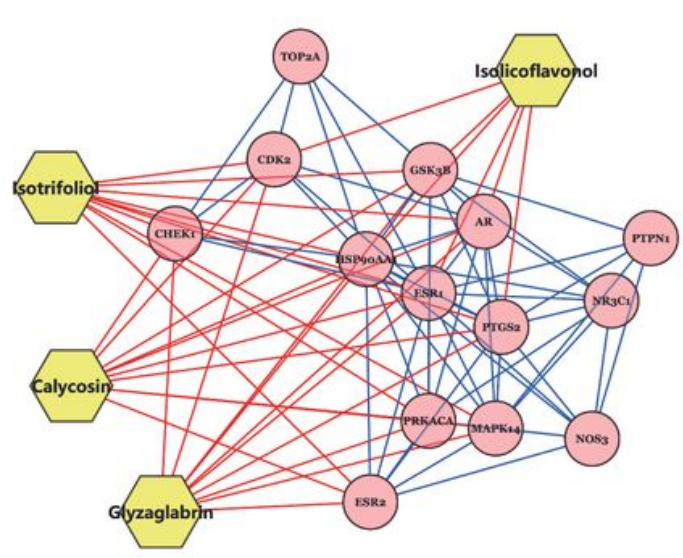

MCODE Score $=10.47118$ Nodes 89 Edges

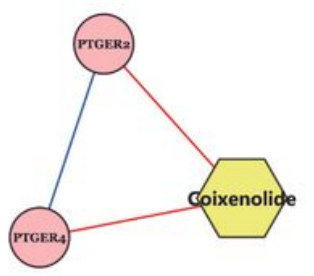

MCODE Score $=3.0003$ Nodes 3 Edges

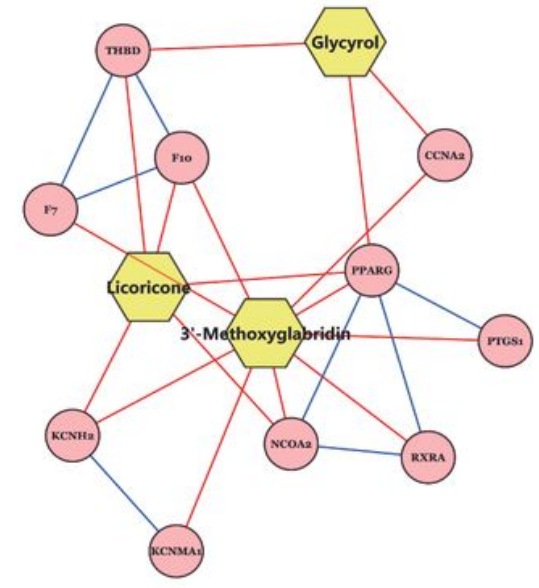

MCODE Score $=4.16713$ Nodes 25 Edges

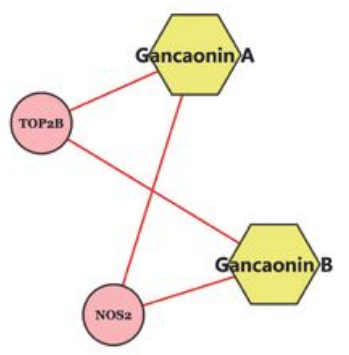

MCODE Score $=2.6674$ Nodes 4 Edges

\section{Figure 6}

Construction of compound-target-function module by MCODE plugin of Cytoscape. Yellow nodes stand for bioactive compounds and pink nodes stand for potential targets. 
A<smiles>COc1ccc2c(=O)c(-c3ccc(OC)c(O)c3)coc2c1</smiles>

B

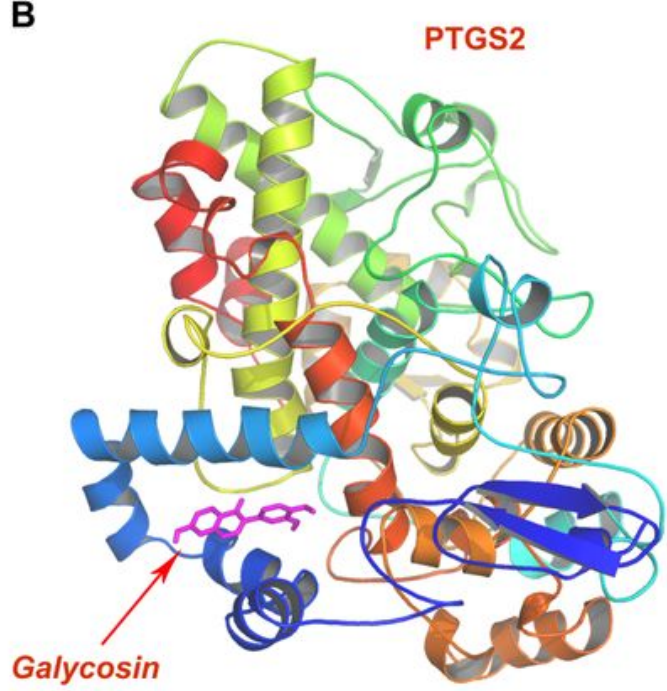

\section{Figure 7}

Molecular docking models of Calycosin binding to PTGS2. (A) Chemical structure of Calycosin. (B) The binding interaction between Calycosin and PTGS2 protein.

\section{Supplementary Files}


This is a list of supplementary files associated with this preprint. Click to download.

- FigureS1.jpg

- TableS1.docx 